\title{
Radial hopper flow prediction using a constitutive model with microstructure evolution
}

\author{
Jin Sun $^{\mathrm{a}}$, Sankaran Sundaresan ${ }^{\mathrm{b}}$ \\ ${ }^{a}$ School of Engineering, University of Edinburgh, Edinburgh EH9 3JL, UK \\ ${ }^{b}$ Department of Chemical and Biological Engineering, Princeton University, \\ Princeton, NJ 08544, USA
}

\begin{abstract}
We present theoretical predictions of granular flow in a conical hopper based on a continuum theory employing a recently-developed constitutive model with microstructure evolution by Sun and Sundaresan [1]. The model is developed for strain rate-independent granular flows. The closures for the pressure and the macroscopic friction coefficient are linked to microstructure through evolution equations for the coordination number and fabric. The material constants in the model are functions of particle-level properties. A salient prediction is the variable stress ratio along the flow direction, in contrast to the constant ratio employed in some widely-used plasticity theories, but supported by results obtained from discrete element simulations. The model permits direct interrogation of the influence of particle-particle friction as well as normal-stress differences on the stress distribution and discharge rate. Increasing particle friction leads to higher stress ratios, but lower normal stress and flow rates, while considering normal-stress differences results in the opposite effects.
\end{abstract}

Keywords:

Granular flow, constitutive model, microstructure evolution, conical hopper

\section{Introduction}

The flow of granular materials in bins and hoppers is of great practical interest in connection with the handling and transportation of bulk solids such as sand, coal, ore and grain. It has been extensively studied for several decades and generated a significant number of publications with earlier 
results summarized in monographs [2, 3], and review articles [4, 5]. With advances in experimental techniques and computational power, recent results using particle image velocimetry (PIV) [6, 7, 8, 9, 10] and discrete element method (DEM) [11, 12, 13, 14, 15, 16, 17] have shed light on flow kinematics and on particle-property effects on the flow behavior. The understanding also drives the development of continuum theories [18, 19, 20], which are necessary for predicting industry-scale hopper flow.

In spite of the extensive research, a continuum model capable of accurately predicting realistic hopper-flow behavior is still under development. For example, the challenges faced in the constitutive modelling of hopper flow were described in [21]; deficiencies of a Mohr-Coulomb plasticity theory were summarized in [18]. The deficiencies of current models are also reflected in the fact that widely different predictions of well-defined silo flow were made using different models [22]. Precisely due to these difficulties, the hopper-flow problem also serves as a stringent benchmark case to test newly-developed theories for granular materials, such as the stochastic flow-rule [18], non-local hypoplasticity [19] and elasto-plastic models [20].

In this paper, we employ a new constitutive model with microstructure evolution [1] to predict flow in a conical hopper. We utilize the radial solution, first presented by Jenike [23], to reduce the number of spatial variables to one by the inherent symmetry. Such a solution, albeit being rather simplified, helps to elucidate the essential capabilities of the constitutive model and prevents obscuring the features by complexities in boundary conditions and numerical methods. We will demonstrate that the model is capable of predicting a varying stress ratio along the flow path, which is realistic but elusive in previous theories. We will show how the particle properties and microstructure are linked to the continuum variables, which enables studying the role of such microscopic parameters at a much lower computational cost. We will also compare the predictions to DEM simulation results and the so-called hour-glass theory (HGT) [24, 25, 3] that is also a radial-flow solution, but employing the Mohr-Coulomb plasticity theory.

The remaining part is organized as follows; the hopper-flow model is described in section 2 detailing the governing equations (2.1) and the constitutive model (2.2). The results from solving the model are presented and analyzed in section 3 , leading to the conclusions and future work in section 4 . Regarding notation, we employ lightface italics for scalars, the boldface regular font for vectors and the San Serif font for the second-order tensors. 


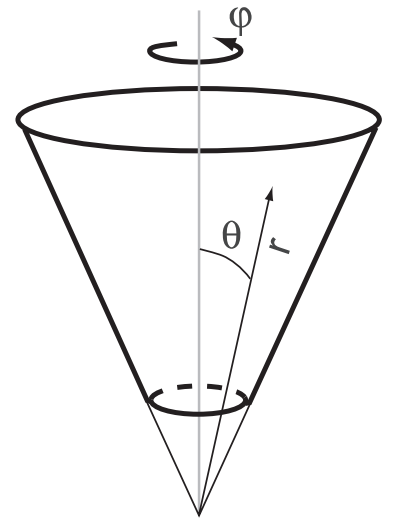

(a)

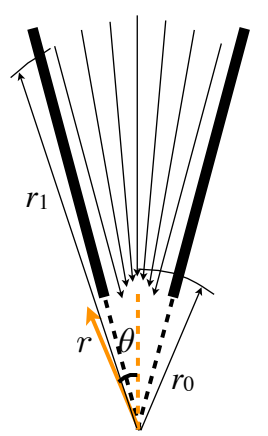

(b)

Figure 1: The schematic diagram of the geometry and the spherical coordinate systems for a conical hopper showing (a) a three-dimensional view and (b) a $r-\theta$ cross-section view with arrows pointing to the virtual apex indicating the radial flow.

\section{Hopper flow modeling}

\subsection{Flow conditions and governing equations}

We consider an incompressible cohesionless granular material of mass density $\rho$ and volume fraction $\phi$ discharging from a narrow-angled conical hopper of half angle $\alpha$, whose geometry is sketched in Fig. 1. We use a spherical coordinate system $((r, \theta, \varphi)$ see Fig. 11(a)) with origin at the virtual apex of the hopper. The walls are assumed to be rigid and smooth. Since the angle $\alpha$ is small, gravity is assumed to be in the radial direction. By axial symmetry, the shear stress $\sigma_{r \theta}$ is reasonably assumed to be zero. We further assume that the velocity is radial and independent of $\theta$ and $\varphi$ coordinates.

With these conditions, the continuity equation for this incompressible granular flow is reduced to

$$
v_{r}=-\frac{V}{r^{2}},
$$

where $V$ is a positive flow rate independent of $r$. This flow rate is related to the discharge mass flow rate $W$, as $W=2 \pi \rho \phi(1-\cos \alpha) V$. We then obtain a dimensionless momentum conservation equation utilizing Eq. 1]

$$
\frac{d \hat{\sigma}_{r r}}{d \hat{r}}=\frac{2 \phi \hat{V}^{2}}{\hat{r}^{5}}+\frac{\hat{\sigma}_{\theta \theta}+\hat{\sigma}_{\varphi \varphi}-2 \hat{\sigma}_{r r}}{\hat{r}}-\phi,
$$


where $r$ is scaled by the radius of a free-fall arch $r_{0}$ (see its location in Fig. 1(b)), i.e. $\hat{r}=r / r_{0}$; and the normal stresses $\hat{\sigma}=\sigma /\left(\rho g r_{0}\right)$ and $\hat{V}=$ $V / \sqrt{r_{0}^{5} g}$. Solution of Eq. 2 with appropriate boundary conditions predicts the radial distributions of velocity and stress as well as the flow rate. However, this equation is not closed and a constitutive model for the stress is necessary. We will present the model employed in this study in the next section.

\subsection{Constitutive model}

We briefly present the constitutive model in its general form for completeness. Readers are referred to [1] for details on the derivation and characteristics of the model. In this model, the stress is expressed as

$$
\boldsymbol{\sigma}=p \mathbf{I}-p \eta \hat{\mathrm{S}}+a_{3} p\left(\mathrm{~A} \hat{\mathrm{S}}+\hat{\mathrm{S}} \mathrm{A}-\frac{2}{3}(\mathrm{~A}: \hat{\mathrm{S}}) \mathrm{I}\right)+a_{4} p\left(\mathrm{~A}-\frac{1}{2}(\mathrm{~A}: \hat{\mathrm{S}}) \hat{\mathrm{S}}\right),
$$

where $p$ is the pressure or mean normal stress, $\hat{S}:=S / \sqrt{\frac{1}{2} D^{T}: D}$ with $S$ and $D$ as the deviatoric and full strain rate tensors, respectively, i.e. $S=D-\frac{1}{3} \operatorname{tr}(D) I$, $\eta$ is a scalar macroscopic friction coefficient and I is the identity tensor. The last two terms in Eq. 3 model the normal stress differences (NSD) in simple shear flow, without which the model predicts equal normal stresses for such flow. The pressure and the macroscopic friction coefficient are linked to coordination number $Z_{2}$ and fabric tensor A using

$$
p d / k=\left(a_{1}+a_{2}|\mathrm{~A}|\right)\left(Z_{2}-Z_{c}\right)^{\alpha},
$$

and

$$
\eta=b_{1}+b_{2} \mathrm{~A}: \hat{\mathrm{S}}
$$

respectively, where $d$ is the particle diameter, $k$ is the particle normal stiffness, and $Z_{c}$ a critical coordination number characterizing jamming. In Eqs. 3 5, the coefficients, $a_{i}, b_{j}(i=1,2,3,4$ and $j=1,2)$ and $\alpha$, are material constants with $a_{1,2}$ and $\alpha$ independent of particle friction coefficient $\mu$ and the rest as a function of $\mu$. Their numerical values are given in Table 1 .

The fabric evolution is modeled as

$$
\AA=c_{1} \mathrm{~S}+c_{2}|\mathrm{D}| \mathrm{A}+c_{3}(\mathrm{~A}: \mathrm{S}) \mathrm{A},
$$

where $\AA=\dot{A}+\mathrm{A} \cdot \mathrm{W}-\mathrm{W} \cdot \mathrm{A}$, with $\mathrm{W}$ as the spin tensor, $\mathrm{W}=\frac{1}{2}\left(\nabla \mathbf{v}-(\nabla \mathbf{v})^{\mathrm{T}}\right)$, and $\dot{A}$ denotes its material time derivative. The coordination-number evolution is given as

$$
\dot{Z}_{2}=d_{1}(\mathrm{~A}: \mathrm{S}-\chi|\mathrm{S}|)+d_{2}|\mathrm{D}|\left(f(\phi)-Z_{2}\right)+d_{3} \operatorname{tr}(\mathrm{D}),
$$




\begin{tabular}{cccccccccc}
\hline \hline & $\alpha$ & $a_{1}$ & $a_{2}$ & $a_{3}$ & $a_{4}$ & $b_{1}$ & $b_{2}$ & $Z_{c}$ & $\phi_{c}$ \\
\hline$x_{1}$ & 2 & 0.0073 & -0.1 & 1.57 & -6 & -0.16 & 1.6 & 1.85 & 0.058 \\
$x_{2}$ & & & & -4.5 & -2 & -6 & -6 & -5 & -5 \\
$x_{3}$ & & & & -1.7 & 6 & 0.16 & -2.9 & 4.15 & 0.582 \\
\hline
\end{tabular}

Table 1: Numerical values of $\alpha, a_{1}, a_{2}$ and $x_{1}-x_{3}$ in the fitting expression $x_{1} e^{x_{2} \mu}+x_{3}$, for the material parameters in the pressure and $\eta$ equations, and the $Z_{2}$ evolution equation [1.

\begin{tabular}{cccccccc}
\hline \hline$c_{1}$ & $c_{2}$ & $c_{3}$ & $d_{1}$ & $d_{2}$ & $d_{3}$ & $\beta_{1}$ & $\beta_{2}$ \\
\hline-0.52 & -2.8 & 100 & -45 & 5.6 & -40 & 7.5 & 0.5 \\
\hline
\end{tabular}

Table 2: Numerical values of the material parameters in the microstructure evolution equations [1].

where $\chi=-\left(c_{2}+\sqrt{c_{2}^{2}-8 c_{1} c_{3}}\right) / 2 c_{3}$ equals $\mathrm{A}: \hat{\mathrm{S}}$ for steady simple shear according to Eq. 6, $f(\phi)=Z_{c}+\beta_{1}\left(\phi-\phi_{c}\right)^{\beta_{2}}$ and $\operatorname{tr}(\mathrm{D})$ is the trace of D. In Eqs. 6 and 7, the coefficients, $c_{i}, d_{i}(i=1,2,3)$ and $\beta_{j}(j=1,2)$, are material constants modeled independent of $\mu$, whose values are listed in Table 2 .

Using the assumptions for radial hopper flow in section 2.1, the strain-rate tensor is diagonal, with $\left[D_{r r}, D_{\theta \theta}, D_{\varphi \varphi}\right]=\left[\frac{d v_{r}}{d r}, \frac{v_{r}}{r}, \frac{v_{r}}{r}\right]$ giving $\hat{\mathbf{S}}=\left[\frac{2}{\sqrt{3}},-\frac{1}{\sqrt{3}},-\frac{1}{\sqrt{3}}\right]$. This together with the fabric Eq. 6 determines that the fabric tensor is also diagonal, i.e. $\mathrm{A}=\left[A_{r r}, A_{\theta \theta}, A_{\varphi \varphi}\right]$, with $A_{\theta \theta}=A_{\varphi \varphi}$. This means $-\frac{1}{2} A_{r r}=$ $A_{\theta \theta}=A_{\varphi \varphi}$ since the fabric tensor is defined as being traceless. The fabric evolution equation is thus simplified to

$$
\frac{d A_{r r}}{d \hat{r}}=-\frac{1}{\hat{r}}\left(2 c_{1}+\sqrt{3} c_{2} A_{r r}+3 c_{3} A_{r r}^{2}\right) .
$$

The boundary condition of $A_{r r}=0$ at the top surface of radius $r_{1}$ (see Fig. 1 (b) for its location) is applied for this equation, which is a reasonable assumption of the isotropic state due to random deposition and lack of substantial shear deformation at the top.

Substituting the fabric and strain-rate tensors in Eq. 5, we obtain

$$
\eta=b_{1}+\sqrt{3} b_{2} A_{r r}
$$


Similarly with the stress Eq. 3, we have the stress components

$$
\begin{aligned}
\sigma_{r r} & =p\left(1-\frac{2}{3} \mu_{b}\right), \\
\sigma_{\theta \theta} & =\sigma_{\varphi \varphi}=p\left(1+\frac{1}{3} \mu_{b}\right),
\end{aligned}
$$

where $\mu_{b}$ is a bulk friction coefficient defined as the ratio of (Von Mises) shear stress to pressure and

$$
\mu_{b}=\sqrt{3}\left(\eta-a_{3} A_{r r}\right) .
$$

The bulk friction coefficient can thus be calculated from fabric and particle friction and need not be taken as a constant as in many plasticity theories. Defining the relation between the stress components as $K \sigma_{r r}=\sigma_{\theta \theta}=\sigma_{\varphi \varphi}$, we then get the stress ratio

$$
K=\frac{1+\frac{1}{3} \mu_{b}}{1-\frac{2}{3} \mu_{b}} .
$$

It can be seen that the relation between the stress components is derived from the stress equation given the fabric and flow conditions. We do not invoke the Haar-von Karman hypothesis [3] to reach this result.

With this relation for stress components, the momentum conservation given in Eq. 2 can be recast as

$$
\frac{d \hat{\sigma}_{r r}}{d \hat{r}}=\frac{2 \phi \hat{V}^{2}}{\hat{r}^{5}}+\frac{2(K-1) \hat{\sigma}_{r r}}{\hat{r}}-\phi,
$$

For this equation, we apply two boundary conditions, assuming a free-fall arch of radius $r_{0}$ and stress-free top surface of radius $r_{1}$. We note that the free-fall arch concept is echoed by the force-chain idea in recent granular material research. Such arch can be seen from the visualization of force chains in the DEM simulation of a flat-bottom silo [13]. Equation 13 is closed by the constitutive relations Eqs. 812 and specific solutions can be obtained applying the boundary conditions. The pressure and $Z_{2}$ evolution equations are redundant for incompressible flow, as evidenced from the above derivation process. 


\section{Hopper flow predictions}

Solving the radial model obtained in section 2.2 for hopper flow of particles with friction $\mu=0.3$, the stress and fabric radial distributions are obtained and shown in Fig. 2. The present theory predicts that the normalstress ratio $K$ increases along the flow direction (see Fig. 2 (a)), which naturally arises from the variation of the fabric (i.e. $A_{r r}$, shown in Fig. 2 (b)). The value $A_{r r}$ increases along the flow, indicating microstructure anisotropy increased due to large deformation. As prescribed in Eq. 9, the increase of $A_{r r}$ results in the increase in $\eta$ along the flow direction, which in turn increases the $K$ values according to Eq. 12, The NSD effect further couples the $A_{r r}$ variation to $K$, reflected in Eq. 11. As seen in Fig. 2 (a), the formulation with the NSD leads to smaller $K$ values, 1.8 at the bottom, comparing to $K=2.1$ calculated without the NSD. In contrast, a constant $K$ is prescribed by the Mohr-Coulomb yield criterion together with the incipientyield-everywhere assumption, which was employed in many previous hopperflow analyses [24, 3, more references therein], and simulations [26]. The essential difference in the present constitutive model is that the $K$ value, a bulk level property, need not be specified a priori; but is calculated from the particle-level property, $\mu$, and the microstructure evolution. Another distinction has to be made from the constant $K$ used in pressure analysis for a static granular column, which appears to be a reasonable assumption in the static condition, as seen in Janssen's analysis [3] and verified more recently by an elasticity theory [27]. This comparison highlights the fact that the stress state in the flow is different from that in the static condition.

To compare these continuum results with DEM ones, we calculate an internal friction coefficient $f$, using the same definition as in a DEM study of two-dimensional hopper flow [12]. This coefficient is related to the stress ratio $K$ as $f=\frac{K-1}{2 \sqrt{K}}$. The value of $f$ increases from 0.121 at the top to 0.382 (0.300) at the bottom for the case without (with) the NSD as plotted in Fig 2(c). This variation agrees with that of $0.150-0.389$ from the DEM simulation for a hopper with $\alpha=30^{\circ}$ discharging polydisperse particles of $\mu=0.3$ [12]. We note that it is reasonable to compare to the polydisperse system simulated as the monodisperse system formed hexagonal crystalline structures [12], which is not consistant with the physical basis of the current constitutive model and not realistic for practical hopper flows. This trend of internal friction increasing from top to bottom is also supported by DEM simulations of cylindrical flat-bottom silos [11, 13, where the ratios between 
the reported shear and normal stresses varied in a similar fashion. However, no quantitative comparison can be made due to the lack of principal stress data and the different geometry used in [11, 13]. With support from the DEM simulations, our results confirm that the new constitutive model has advantages in predicting realistic characteristics of stress in the conical hopper flow.

Predictions of normal stress $\hat{\sigma}_{r r}$ from the present formulations with and without the NSD effect and from the HGT are compared in Fig. 2 (d). The HGT uses a constant $K$ equal to the mean of the $K$ values from the calculation with NSD. The profiles of $\hat{\sigma}_{r r}$ along the radial distance $\hat{r}$ are similar, with the HGT result being the largest and reaching the peak value at the lowest position. The differences in the normal stress profiles also lead to different predictions of the flow rate. The values of $\hat{V}$ from the calculations without NSD, with NSD and using HGT are 1.550, 1.867 and 1.992, respectively, which shows that larger $\hat{\sigma}_{r r}$ results in a higher flow rate. To put this flow rate in perspective to commonly measured discharge rates, we show that the Beverloo equation in the form of $W=C \rho \phi \sqrt{g(D-k d)^{5}}$ [28, 4], where $\mathrm{D}$ is the aperture diameter and $k$ is of order unity, can be related to $\hat{V}$ as $C=\hat{V} \pi /(4 \sqrt{2 \sin \alpha})$. The $C$ measured in experiments takes a value close to 0.58 [4]. However, according to the predictions of $\hat{V}, C$ is at least 1.22 , about twice of the common value for a $\alpha=30^{\circ}$ hopper. This over-prediction should mostly be ascribed to the radial analysis itself, not the constitutive model though. One major source of error is the assumption of smooth wall in this analysis. Because of this gross over-prediction, we shall not assess the capability of the three different analyses regarding discharge rate prediction. As a summary of the NSD effect, with NSD the predictions of the normal stress $\hat{\sigma}_{r r}$ and the flow rate are higher, but the stress ratio $K$ is lower, than without NSD.

Another important functionality of the constitutive model used in this study is to directly probe the particle-friction effect on flow behavior. We have performed the same hopper-flow analysis for granular materials with particle friction $\mu=0.1$ and $\mu=1.0$. The results for $K$ and $\hat{\sigma}_{r r}$ are plotted in Figs. 3 (a)-(b), and (c)-(d), respectively, of which (a)-(c) are for $\mu=0.1$ and (b)-(d) for $\mu=1.0$. The fabric is modeled as independent of $\mu$ and thus not repeated in this figure. Comparing Figs. 3 (a) and (b) together with Fig. 2 (a), it can be seen that increasing $\mu$ results in higher $K$ values. The difference between predictions with and without NSD is also enlarged. 


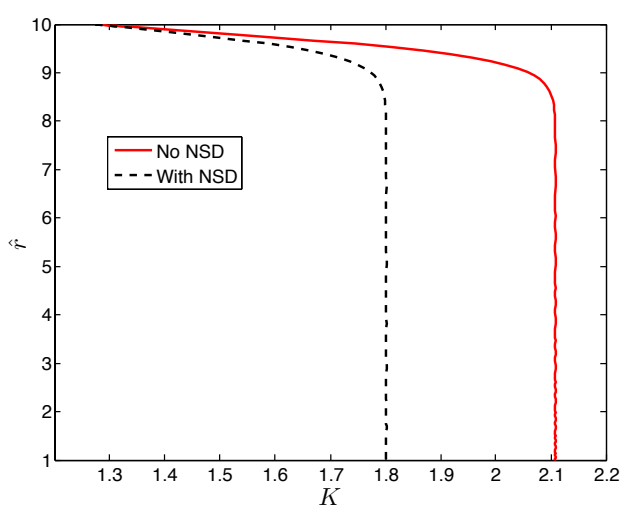

(a)

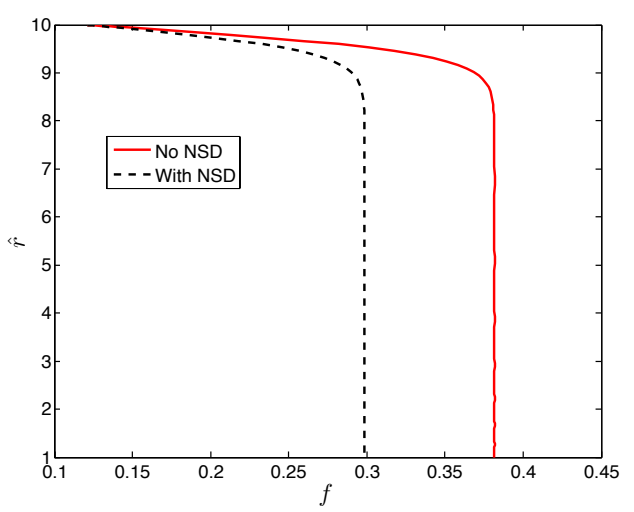

(c)

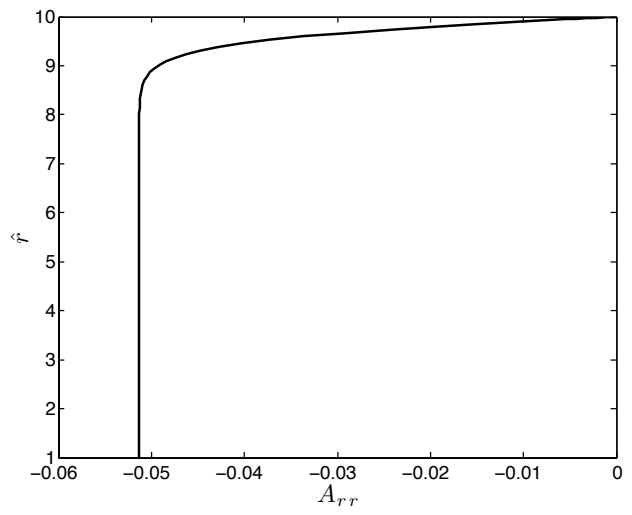

(b)

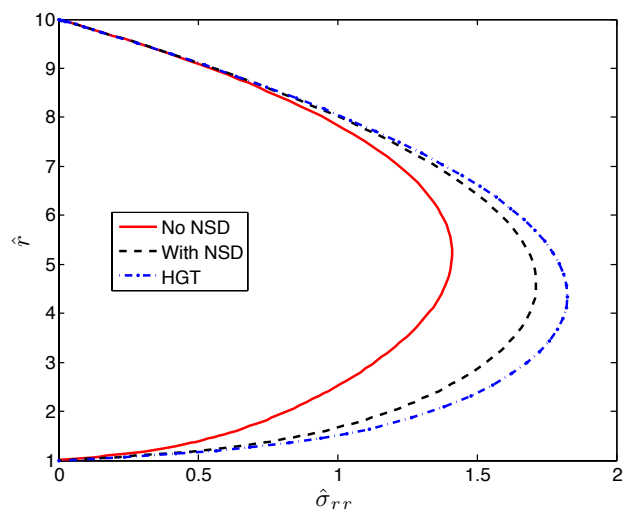

(d)

Figure 2: Radial variation of (a)stress ratio, (b) component of the fabric tensor, (c) macroscopic friction coefficient and (d) normal stress, plotted against the dimensionless distance in the vertical axis. The material has particle friction $\mu=0.3$. 
Similar trend is observed for the internal friction coefficient $f$ (not shown), which is consistent with engineering observations although no quantitative comparison can be made at the moment. Studying the $\hat{\sigma}_{r r}$ in Figs. 3 (c)-(d) and Fig. 2 (d), it can be concluded that higher $\mu$ leads to lower normal stress and does not alter the effect of NSD. From the flow-rate analysis above, it can also be deduced that higher $\mu$ results in lower flow rates. Specifically, the $\hat{V}$ values from the calculations without NSD, with NSD, and using HGT equal $2.161,2.403$ and 2.545 , respectively, when $\mu=0.1$; and $1.365,1.718$ and 1.83 , respectively, when $\mu=1.0$. This effect of particle friction qualitatively matches practical experience and is supported by the DEM simulations of a cylindrical silo [16], but again quantitative comparison cannot be made due to the lack of directly comparable data. The increase in friction also amplifies the difference between the flow rate predictions for cases with and without NSD from about $11 \%$ to $26 \%$. However, the potential of the constitutive model in facilitating understanding of the particle-property influence on the stress distribution and flow rate can clearly be seen from the above results.

\section{Conclusions}

A radial-flow solution of the conical hopper flow has been carried out utilizing a recently-developed constitutive model [1]. Results on stress, microstructure and flow rate have been obtained by simultaneously solving the continuity, momentum and fabric evolution equations. It has been demonstrated that a varying stress ratio is predicted, as a result of the fabric evolution. The particle friction has been shown to lead to higher stress ratios, but lower normal stress and flow rates as it increases. These results are supported qualitatively and quantitatively by DEM simulations and engineering observations. The effects of considering the NSD, have been explored, showing that considering NSD results in higher normal stress and flow rate, but lower stress ratio. The difference can be as large as about 30\%, indicating that the NSD in constitutive models warrants more attention in order to make more accurate predictions.

The significance of the constitutive model has been demonstrated to lie in connecting particle-level properties to continuum-level computation through microstructure evolution, obviating the need for specifying bulk properties and enabling prediction of particle-property effects on flow. Admittedly, the radial analysis performed is too simplified to validate against other spatial measurements, or to be used as a predictive tool for hopper design. Fu- 


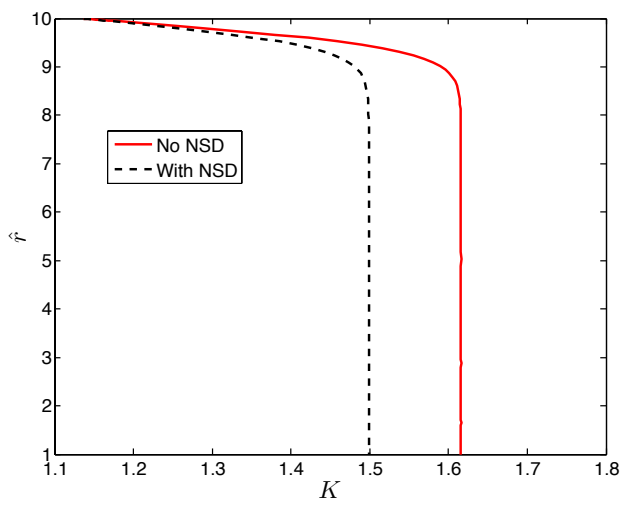

(a)

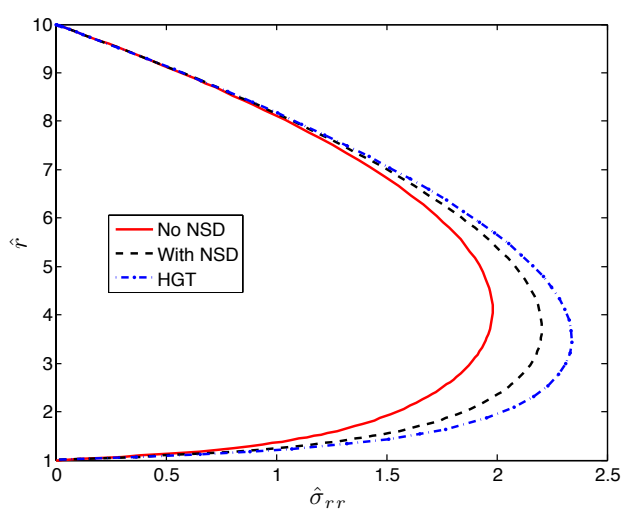

(c)

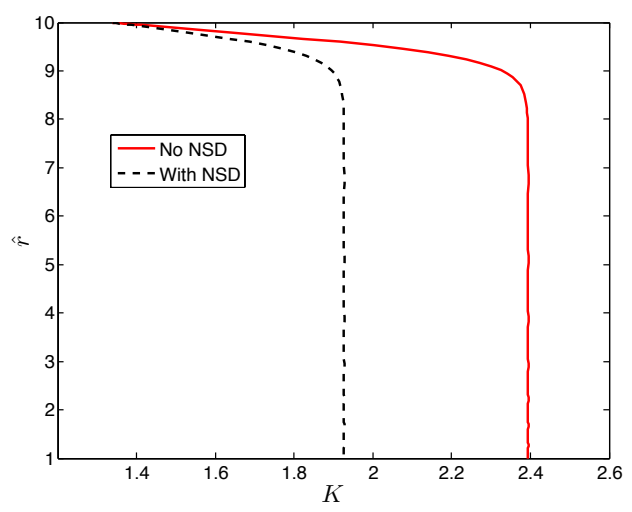

(b)

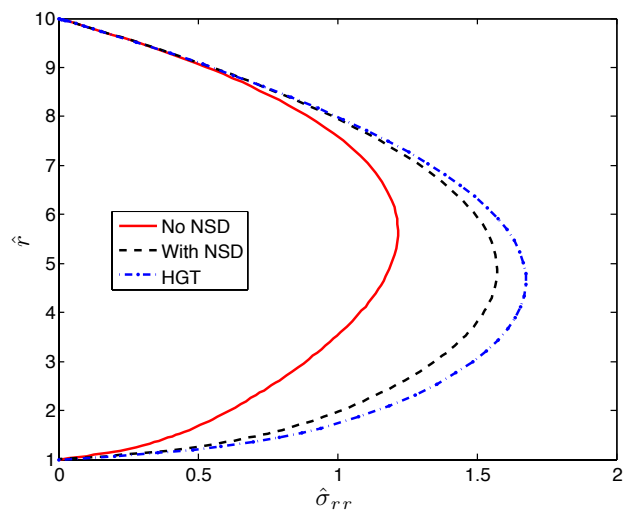

(d)

Figure 3: Radial variation of stress ratio in (a) and (b), of normal stress in (c) and (d), plotted against the dimensionless distance in the vertical axis. The particle-friction coefficient is $\mu=0.1$ for (a) and (c), and $\mu=1.0$ for (b) and (d). 
ture work has been planned on applying the constitutive model to multidimensional analysis of compressible flow and validating against salient hopper flow behavior, such as shear localization.

\section{Acknowledgment}

The authors would like to acknowledge the support from the Department of Energy National Energy Technology Laboratory under grants DE-FG2607NT43070 and de-fe0006932.

\section{References}

[1] J. Sun, S. Sundaresan, A constitutive model with microstructure evolution for flow of rate-independent granular materials, Journal of Fluid Mechanics 682 (2011) 590-616.

[2] A. Drescher, Analytical methods in bin-load analysis, Elsevier, New York, 1991.

[3] R. M. Nedderman, Statics and Kinematics of Granular Materials, Cambridge University Press, 1992.

[4] R. M. Nedderman, U. Tüzün, S. B. Savage, G. T. Houlsby, The flow of granular materials-I: Discharge rates from hoppers, Chemical Engineering Science 37 (1982) 1597-1609.

[5] U. Tüzün, G. T. Houlsby, R. M. Nedderman, S. B. Savage, The flow of granular materials-II velocity distributions in slow flow, Chemical Engineering Science 37 (1982) 1691-1709.

[6] P. A. Langston, M. S. Nikitidis, U. Tüzün, D. M. Heyes, N. M. Spyrou, Microstructural simulation and imaging of granular flows in two- and three-dimensional hoppers, Powder Technology 94 (1997) 59-72.

[7] J. U. Böhrnsen, H. Antes, M. Ostendorf, J. Schwedes, Silo discharge: Measurement and simulation of dynamic behavior in bulk solids, Chemical Engineering \& Technology 27 (2004) 71-76.

[8] J. Choi, A. Kudrolli, M. Z. Bazant, Velocity profile of granular flows inside silos and hoppers, Journal of Physics: Condensed Matter 17 (2005) S2533-S2548. 
[9] A. Garcimartín, I. Zuriguel, A. Janda, D. Maza, Fluctuations of grains inside a discharging two-dimensional silo, Physical Review E 84 (2011) 031309 .

[10] I. Sielamowicz, M. Czech, T. A. Kowalewski, Empirical analysis of eccentric flow registered by the dpiv technique inside a silo model, Powder Technology 212 (2011) 38-56.

[11] P. A. Langston, U. Tüzün, D. M. Heyes, Discrete element simulation of internal stress and flow fields in funnel flow hoppers, Powder Technology 85 (1995) 153-169.

[12] A. V. Potapov, C. S. Campbell, Computer simulation of hopper flow, Physics of Fluids 8 (1996) 2884-2894.

[13] H. P. Zhu, A. B. Yu, Y. H. Wu, Numerical investigation of steady and unsteady state hopper flows, Powder Technology 170 (2006) 125-134.

[14] W. R. Ketterhagen, J. S. Curtis, C. R. Wassgren, B. C. Hancock, Predicting the flow mode from hoppers using the discrete element method, Powder Technology 195 (2009) 1-10.

[15] C. H. Rycroft, K. Kamrin, M. Z. Bazant, Assessing continuum postulates in simulations of granular flow, Journal of the Mechanics and Physics of Solids 57 (2009) 828-839.

[16] J. E. Hilton, P. W. Cleary, The effect of rotational shear on granular discharge rates, Physics of Fluids 22 (2010) 071701.

[17] R. Balevičius, I. Sielamowicz, Z. Mróz, R. Kačianauskas, Investigation of wall stress and outflow rate in a flat-bottomed bin: A comparison of the dem model results with the experimental measurements, Powder Technology 214 (2011) 322-336.

[18] K. Kamrin, M. Z. Bazant, Stochastic flow rule for granular materials, Physical Review E 75 (2007) 041301-28.

[19] M. Wójcik, J. Tejchman, Modeling of shear localization during confined granular flow in silos within non-local hypoplasticity, Powder Technology 192 (2009) 298-310. 
[20] K. Kamrin, Nonlinear elasto-plastic model for dense granular flow, International Journal of Plasticity 26 (2010) 167-188.

[21] A. Drescher, Some aspects of flow of granular materials in hoppers, Philosophical Transactions: Mathematical, Physical and Engineering Sciences 356 (1998) 2649-2666.

[22] J. M. Rotter, J. M. F. G. Holst, J. Y. Ooi, A. M. Sanad, Silo pressure predictions using discrete-element and finite-element analyses, Philosophical Transactions: Mathematical, Physical and Engineering Sciences 356 (1998) 2685-2712.

[23] A. W. Jenike, Gravity Flows of Bulk Solids, Bulletin 108 Vol. 52, University of Utah, Salt Lake City, 1961.

[24] S. B. Savage, M. Sayed, Gravity flow of coarse cohesionless granular materials in conical hoppers, Zeitschrift für Angewandte Mathematik und Physik (ZAMP) 32 (1981) 125-143.

[25] R. Jackson, Some mathematical and physical aspects of continuum models for the motion of granular materials, in: R. E. Meyer (Ed.), Theory of Dispersed Multiphase Flow, Academic Press, San Diego, 1983, pp. 291-337.

[26] P. A. Gremaud, J. V. Matthews, On the computation of steady hopper flows: I. stress determination for coulomb materials, Journal of Computational Physics 166 (2001) 63-83.

[27] K. Bräuer, M. Pfitzner, D. O. Krimer, M. Mayer, Y. Jiang, M. Liu, Granular elasticity: Stress distributions in silos and under point loads, Physical Review E 74 (2006) 061311-10.

[28] W. Beverloo, H. Leniger, J. Van de Velde, The flow of granular material through orifices, Journal of Chemical Science 15 (1961). 